\title{
Afterglow Effects as a Tool to Screen Emissive Non-Geminate Charge Recombination Processes in Organic Photovoltaic Composites
}

Panagiotis E. Keivanidis ${ }^{1,2}{ }^{*}$, Grigorios Itskos $^{3}$, Zhipeng Kan $^{2}{ }^{2}$,Eduardo Aluicio-Sarduy ${ }^{2}$, Hossein Goudarzi $^{2}$, Valentin Kamm ${ }^{4}$, Frédéric Laquai ${ }^{5}$, Weimin Zhang ${ }^{5,}{ }^{6}$, Christoph Brabec ${ }^{7,8}$, George Floudas $^{4,9}$, Iain McCulloch ${ }^{5,6}$

${ }^{1}$ Device Technology and Chemical Physics Lab, Department of Mechanical Engineering and Materials Science and Engineering, Cyprus University of Technology, Limassol 3041, Cyprus

${ }^{2}$ Centre for Nano Science and Technology @ PoliMi, Fondazione Istituto Italiano di Tecnologia, Via Pascoli 70/3, 20133 Milano, Italy

${ }^{3}$ Department of Physics, Experimental Condensed Matter Physics Laboratory, University of Cyprus, Nicosia 1678, Cyprus

${ }^{4}$ Max Planck Institute for Polymer Research, Ackermannweg 10, D-55128, Mainz, Germany

${ }^{5}$ King Abdullah University of Science and Technology (KAUST), KAUST Solar Center (KSC), Physical Sciences and Engineering Division (PSE), Thuwal 23955-6900, Kingdom of Saudi Arabia

${ }^{6}$ Department of Chemistry and Centre for Plastic Electronics, Imperial College London, London SW7 2AZ, United Kingdom

${ }^{7}$ Institute of Materials for Electronics and Energy Technology (I-MEET), Friedrich-AlexanderUniversity Erlangen-Nuremberg, Martensstraße 7, 91058 Erlangen, Germany

${ }^{8}$ Bavarian Center for Applied Energy Research (ZAE Bayern), Haberstrasse 2a, 91058 Erlangen, Germany

${ }^{9}$ University of Ioannina, Department of Physics, 45110 Ioannina, Greece

* Corresponding Author: p.keivanidis@ cut.ac.cy

Keywords: solar cell; photodetector; perylene diimides; fill factor; charge trapping; delayed luminescence; multiple-diode equivalent circuit; non-fullerene acceptors 


\begin{abstract}
Disentangling temporally-overlapping charge carrier recombination events in organic bulk heterojunctions by optical spectroscopy is challenging. Here, a new methodology for employing delayed luminescence spectroscopy is presented. The proposed method is capable of distinguishing between recombination of spatially-separated charge carriers and trap-assisted charge recombination simply by monitoring the delayed luminescence (afterglow) of bulk heterojunctions with a quasi timeintegrated detection scheme. Applied on the model composite of the donor poly(6,12-dihydro6,6,12,12-tetraoctyl-indeno[1,2-b]fluorene-alt-benzothiadiazole) (PIF8BT) polymer and the acceptor ethyl-propyl perylene diimide (PDI) derivative, i.e. PIF8BT:PDI, the luminescence of charge-transfer (CT) states created by non-geminate charge recombination on the ns - $\mu$ s time scale is observed. Fluence-dependent, quasi time-integrated detection of the CT luminescence monitors exclusively emissive charge recombination events, while rejecting the contribution of other early-time emissive processes. Trap-assisted and bimolecular charge recombination channels are identified based on their distinct dependence on fluence. The importance of the two recombination channels is correlated with the layer's order and electrical properties of the corresponding devices. Four different microstructures of the PIF8BT:PDI composite obtained by thermal annealing are investigated. Thermal annealing of PIF8BT:PDI shrinks the PDI domains in parallel with the growth of the PIF8BT domains in the blend. Common to all states studied, the delayed CT luminescence signal is dominated by trap-assisted recombination. Yet, the minor fraction of fully-separated charge recombination in the overall CT emission increases as the difference in the size of the donor and acceptor domains in the PIF8BT:PDI blend becomes larger. Electric field-induced quenching measurements on complete PIF8BT:PDI devices confirm quantitatively the dominance of emissive trap-limited charge recombination and demonstrate that only $40 \%$ of the PIF8BT/PDI CT luminescence comes from the recombination of fully-separated charges, taking place within $200 \mathrm{~ns}$ after photoexcitation. The method is applicable to other non-fullerene acceptor blends beyond the system discussed here, if their CT state luminescence can be monitored.
\end{abstract}




\section{Introduction}

Bulk heterojunction organic photovoltaic (OPV) devices ${ }^{1,2}$ offer a promising route towards fabrication of low-cost solar cells of high efficiency, ${ }^{3}$ capable to support several technologically important applications including building integrated photovoltaics, ${ }^{4}$ indoor power generation for portable devices, ${ }^{5}$ and low-power driven wearable electronic circuits. ${ }^{6}$

Typical OPV device geometries comprise thin photoactive layers of blend systems prepared by electron donating polymeric matrices mixed with electron accepting molecules. ${ }^{7}$ Following light absorption by the OPV film, photo-induced charge transfer (CT) reactions at a large number of donor/acceptor interfaces facilitate the formation of electrostatically correlated electron-hole pairs, most widely defined as interfacial CT states. ${ }^{8}$ For several OPV material combinations, the spectroscopic signature of interfacial CT states is the appearance of a typical exciplex photoluminescence (PL) signal that is driven by the recombination of geminate charge pairs, and that is spectrally red shifted with respect to the luminescence signal of the OPV components alone. ${ }^{9}$ Alternatively, the complete separation of the geminate charge pairs quenches the exciplex luminescence, thereby leading to the creation of free carriers that can be extracted at the device electrodes. Apart from providing evidence for the efficient generation of electron-hole pairs, the PL signal of the CT state serve as a marker for the maximum open circuit voltage $\left(V_{o c}\right)$ that can be obtained by the OPV combination under study. ${ }^{10,11}$

In both processes of charge pair generation and full separation, the impact of layer order holds a major key-role; the optimized heterojunction microstructure ${ }^{12}$ of the donor/acceptor interface not only affects the efficiency of generation and dissociation of the interfacial CT state at early time scales after light absorption, but it also favours free charge recombination at much longer time delays when charge carrier mobility is low or when charges become trapped at structural defects present in the OPV layer. Ideally, a bi-continuous network of coarsened, well-ordered donor and acceptor phases in the bulk heterojunction film is required to support the dissociation of the promptly formed interfacial CT states 
and to assist the transport of free carriers towards the device contacts. ${ }^{13-16}$ In practice, however, the performance of OPV devices is limited by factors linked to inappropriate heterojunction morphologies that either promote the recombination of geminate charges, or impede charge transport and increase the probability for free charge recombination at the expense of charge extraction efficiency. ${ }^{17-21}$ In addition, owing to the limited order of OPV bulk heterojunction layers, a large concentration of structural defects enhances the losses caused by the trap-limited charge recombination route. ${ }^{22,23}$ Another important loss channel is the recombination of free charge carriers that proceeds via the formation of an encounter complex ${ }^{24}$ and that activates the interfacial CT states at longer time scales. Both types of non-geminate charge recombination can sometimes be emissive manifesting in the activation of $\mu$ s time-scale delayed CT luminescence of the composite. ${ }^{11,25}$ It is not surprising that well-ordered donor/acceptor interfaces facilitate a high photocurrent generation efficiency for devices, but they can also enable a strong delayed CT luminescence. ${ }^{26}$ Throughout the text below the term nongeminate recombination will be used for generally referring to the processes of trap-limited and free charge recombination.

In optical spectroscopy, the phenomenon of afterglow is widely known from the area of inorganic phosphors. ${ }^{27}$ Afterglow effects correspond to the persistent luminescence originating from impurities in the material structure that act as trap-sites. ${ }^{28}$ Afterglow effects can also be utilized in organic material structures for developing photonic devices. ${ }^{29,} 30$ The lifetime of charge carriers in some organic materials may be in the order of 2 hours, ${ }^{31}$ therefore the spectroscopic study of organic afterglow can become a valuable tool for monitoring the decay pathways of photogenerated charges in photoactive composites.

In most of the developed OPV systems, the decay pathways of charges are activated by geminate and trap-limited charge recombination processes and are responsible for losses in the short-circuit photocurrent. Notably, these losses are mainly linked to OPV device platforms that are best described 
by a single-diode equivalent circuit diagram. ${ }^{32}$ Conversely, the recombination of free charges (bimolecular) leads to the reduction of $V_{o c}$ in the OPV device.

The minimization of the recombination losses in OPV systems is of paramount importance for the development of high-performance OPV devices. ${ }^{33}$ To this end, non-geminate charge recombination can be monitored by a limited number of experimental techniques such as transient absorption, ${ }^{34}$ timedelayed collection-field, intensity modulated photocurrent/photovoltage spectroscopy, ${ }^{35}$ microwave resonance, ${ }^{36}$ time-resolved microwave conductance and photo-CELIV (charge extraction by linearly increasing voltage). ${ }^{24,}{ }^{37}$ Especially for the case of new emerging molecular structures of non-fullerene based electron acceptors, non-geminate charge recombination losses seem to dictate the overall performance of the corresponding OPV devices therefore the detailed study of this particular loss channel should be further pursued by the broader community of OPV technologists. To enable the identification of the optimum material combinations for the plethora of materials generated, more experimental tools are sought to support the fast material screening in a straightforward and reliable manner.

Here we present a new a methodology of material screening that utilizes the spectroscopic technique of delayed luminescence for discriminating trap-assisted and bimolecular charge recombination in OPV composite films. We adapt an experimental characterization approach whereby the two different types of non-geminate charge recombination that occur on comparable time scales can be well resolved with the application of quasi steady-state afterglow detection scheme as a function of photoexcitation intensity. The applicability of the proposed methodology is demonstrated by studying the fullerenefree OPV bulk heterojunction system comprising the poly(6,12-dihydro-6,6,12,12-tetraoctylindeno[1,2-b]fluorene-alt-benzothiadiazole) (PIF8BT) copolymer mixed with the ethyl-propyl perylene diimide (PDI) derivative. The PIF8BT:PDI composite is an ideal model system due to the capability of its thin films to exhibit CT afterglow driven by emissive non-geminate recombination. ${ }^{25}$, ${ }^{26}$ Our proposed quasi steady-state afterglow detection scheme is discussed in light of detailed 
information on the structural motifs of the PIF8BT:PDI blend, and the electrical properties of the corresponding OPV devices. As such, a rigorous correlation between the delayed luminescence detection, layer order and device performance is established that can be of great value in the effort to understand similar OPV composite systems.

\section{Experimental}

Materials: The protocol for the synthesis of PIF8BT (batch code WZ/08/023) has been described elsewhere. ${ }^{25}$ PDI and poly(styrene) (PS) were purchased from Solarmer and Sigma-Aldrich respectively and used as received.

Thin film preparation: PIF8BT:PDI and PS:PDI solutions were prepared in chloroform with a 18.75 $\mathrm{mg} / \mathrm{ml}$ PIF8BT mass concentration, and with a 2:3 PIF8BT:PDI composition ratio. Solutions were spun at $3000 \mathrm{rpm}$ for $1 \mathrm{~min}$ onto pre-cleaned fused silica quartz (spectrosil B) substrates and thermal annealing was performed in a range of temperatures between $50-150{ }^{\circ} \mathrm{C}$ for $15 \mathrm{~min}$. For all layers, film development was performed in a $\mathrm{N}_{2}$-filled glovebox and the thickness of the obtained films was kept in the range of $100 \mathrm{~nm}$, as determined by a Dektak profilometer (Bruker, D150).

UV-Vis and photoluminescence characterization: UV-vis absorption and PL spectra of the fabricated films were recorded with UV-2700 Shimadzu spectrophotometer and a Horiba Jobin Yvon NanoLog spectrofluorimeter, respectively.

Device fabrication: All PIF8BT:PDI and PS:PDI devices were fabricated by using commercially available ITO substrates (XY15S, surface polished glass/Indium Tin Oxide substrates from Xin Yan Technology Ltd.). The ITO substrate was ultrasonically cleaned using acetone and isopropanol for 15 min. After preliminary cleaning, the substrate was washed with Hellmanex III to remove contaminants and residues from the ITO surface. The substrate was again cleaned with DI water followed by acetone and isopropanol for $15 \mathrm{~min}$ and soon after placed in oxygen plasma (100 W power) for $10 \mathrm{~min}$. For conventional device geometry samples with the glass/ITO/PEDOT:PSS/polymer:PDI/Al 
configuration, a PEDOT:PSS hole-collecting layer was deposited on the glass/ITO electrodes by spincoating and it was annealed at $140{ }^{\circ} \mathrm{C}$ for $30 \mathrm{~min}$. Then the substrates were transferred in a $\mathrm{N}_{2}$-filled glovebox and the photoactive layers (PIF8BT:PDI or PS:PDI) were spun on the PEDOT:PSS layer. The deposition of the Al metal electrode was enabled thermal evaporation in vacuum $\left(1 \times 10^{-6} \mathrm{mbar}\right)$ on top of the photoactive layers. For inverted device geometry samples with the glass/ITO/zinc oxide ( $\mathrm{ZnO}) / \mathrm{PIF} 8 \mathrm{BT}: \mathrm{PDI} / \mathrm{V}_{2} \mathrm{O}_{5} / \mathrm{Ag}$ configuration, a $\mathrm{ZnO}$ electron-collecting layer was deposited on the glass/ITO substrate by spin-coating and it was annealed at $140{ }^{\circ} \mathrm{C}$ for $30 \mathrm{~min}$. Then the substrates were transferred in a $\mathrm{N}_{2}$-filled glovebox and the PIF8BT:PDI photoactive layer were spun on $\mathrm{ZnO}$. The deposition of the $\mathrm{V}_{2} \mathrm{O}_{5}$ interlayer and the $\mathrm{Ag}$ electrode was performed by thermal evaporation in vacuum $\left(1 \times 10^{-6}\right.$ mbar $)$ on top of a photoactive film. Annealing of the devices was performed after completing top electrode deposition. Both conventional and inverted device geometry devices were encapsulated with degassed epoxy and glass slide of $1 \mathrm{~mm}$ thickness in the glovebox.

Photovoltaic performance and external quantum efficiency characterization: The photovoltaic performance of the fabricated solar cells was studied by recording the $\mathrm{J}-\mathrm{V}$ metrics of the devices under simulated solar light (AM1.5G, $78 \mathrm{~mW} \mathrm{~cm}^{-2}$ irradiance), and external quantum efficiency (EQE) was determined with monochromatic illumination.

Space charge limited current device characterization: Unipolar devices were fabricated by keeping the same protocol used for the PIF8BT:PDI photoactive layer preparation. Hole-only devices were prepared based on the glass/ITO/PEDOT:PSS/PIF8BT-PDI/Au configuration while the device configuration of the electron-only devices was glass/ITO/ZnO/PIF8BT-PDI/Ca/Al

Intensity dependent photocurrent and photovoltage device characterization: A diode pumped solidstate cw-laser (DPGL2010F, Lambda Photometrics) was used for photoexciting a set of encapsulated PIF8BT:PDI devices with photoactive layers annealed at different temperatures at $532 \mathrm{~nm}$. The laserinduced short-circuit photocurrent and open-circuit voltage of the devices were recorded with a Keithley electrometer (Source Meter Unit 2401). The laser output (10 mW, $1 \mathrm{~mm}$ diameter) was 
progressively attenuated with the use of combined neutral density filters of known transmittance values at the photoexcitation wavelength. All measurements were performed in ambient, and excellent reproducibility of the measured photocurrent was found.

Atomic force microscopy characterization: The surface topography of the developed PIF8BT:PDI composite films was studied by atomic force microscopy using an Agilent 5500 in tapping mode under ambient conditions. Topography and phase images were recorded simultaneously.

2-D wide angle $x$-ray scattering characterization: Wide-angle X-ray scattering measurements were carried out with a Rigaku MicroMax 007 rotating anode X-ray generator with a maximum power of $800 \mathrm{~W}$ and brightness of $18 \mathrm{~kW} / \mathrm{mm}^{2}$ (operated at a tube voltage of $40 \mathrm{kV}$ and tube current of $\mathrm{I}=12$ mA $(480 \mathrm{~W}))$ utilizing a $\mathrm{Cu}$ target. The detection system was a MAR345 image plate area detector, and the sample-to-detector distance was set at $32.5 \mathrm{~cm}$. Extruded fibers (extrusion temperature $\sim 100$ $\left.{ }^{\circ} \mathrm{C}\right)$ were prepared using a microextruder following cooling to ambient temperature. Subsequently, the filaments were inserted in a capillary and heated from 30 to $200{ }^{\circ} \mathrm{C}$, in $10{ }^{\circ} \mathrm{C}$ steps. At every temperature the diffraction pattern was recorded for $1 \mathrm{~h}$ following a $30 \mathrm{~min}$ stabilization interval. From the recorded 2-D diffraction patterns the intensity distributions were obtained and presented as a function of the modulus of the scattering vector $q[q=(4 \pi / \lambda) \sin (2 \theta / 2)$, where $2 \theta$ is the scattering angle]. Domain sizes were determined from the peak full-width at half-maximum (FWHM).

Time-integrated and time-gated gated delayed luminescence characterization of films and devices: Time-integrated and time-gated spectra of the studied systems were recorded at room temperature. The samples were photoexcited with the output of an optical parametric oscillator (Spectra-Physics VersaScan Midband 120) pumped by the third harmonic of a Nd:YAG Laser (Spectra-Physics INDI40-10-HG) at $530 \mathrm{~nm}$ by a train of $10 \mathrm{~ns}$ pulses at a repetition rate of $10 \mathrm{~Hz}$. No focal lens was used for the photoexcitation of the samples, and a laser spot size of $0.385 \mathrm{~cm}^{2}$ was kept for all samples measured. The emitted light was dispersed in a spectrograph (Andor Shamrock Spectrograph, SR303i) with a 150 lines per millimeter grating and detected with a gated intensified charge-coupled device 
camera (Andor iCCD, iStar DH320T-25U-73). Intensity dependent time-gated kinetics were acquired by keeping the delay-step and gate-width values fixed at $500 \mathrm{~ns}$. Quasi-cw delayed luminescence characterization was enabled by a delay-step of $50 \mathrm{~ns}$ and a gate-width of $1 \mathrm{~ms}$. During the spectroscopic characterization, the samples were kept in a liquid-nitrogen variable-temperature cryostat (Janis VPF-100) that was evacuated by a turbomolecular pump (Pfeiffer TSH 071E Economy Dry Vacuum Pumping Station) so that a dynamic vacuum of a typical pressure of $10^{-7}$ mbar was maintained during the measurements. Photoexcitation intensity-dependent measurements were performed by using the combination of a set of neutral density filters of known transmittance values at $532 \mathrm{~nm}$. A DC power supply, (Agilent, E3617A 60W, 60V, 1A) was used for biasing the PIF8BT:PDI and PS:PDI devices in a range of different voltages during the spectroscopic characterization, thereby enabling electric-field induced quenching experiments of delayed luminescence in operative PIF8BT:PDI and PS:PDI photodiode devices.

\section{Results}

The study of the PIF8BT:PDI system was performed with a series of spectroscopic and structural characterization techniques involving UV-Vis absorption, PL, atomic force microscopy (AFM) and wide-angle X-ray scattering (WAXS) measurements, in combination with the electrical characterization of the corresponding PIF8BT:PDI devices. In all cases the content of PDI in the blend was kept to $60 \mathrm{wt} \% .{ }^{25,26}$ The chemical structure of the materials studied, and their frontier orbital alignment ${ }^{25}$ is presented in Figure 1a.

\section{Optical and electrical characterization}

Figure $1 \mathrm{~b}$ presents the UV-Vis absorption spectra for a set of PIF8BT:PDI blends processed at different thermal annealing protocols. 

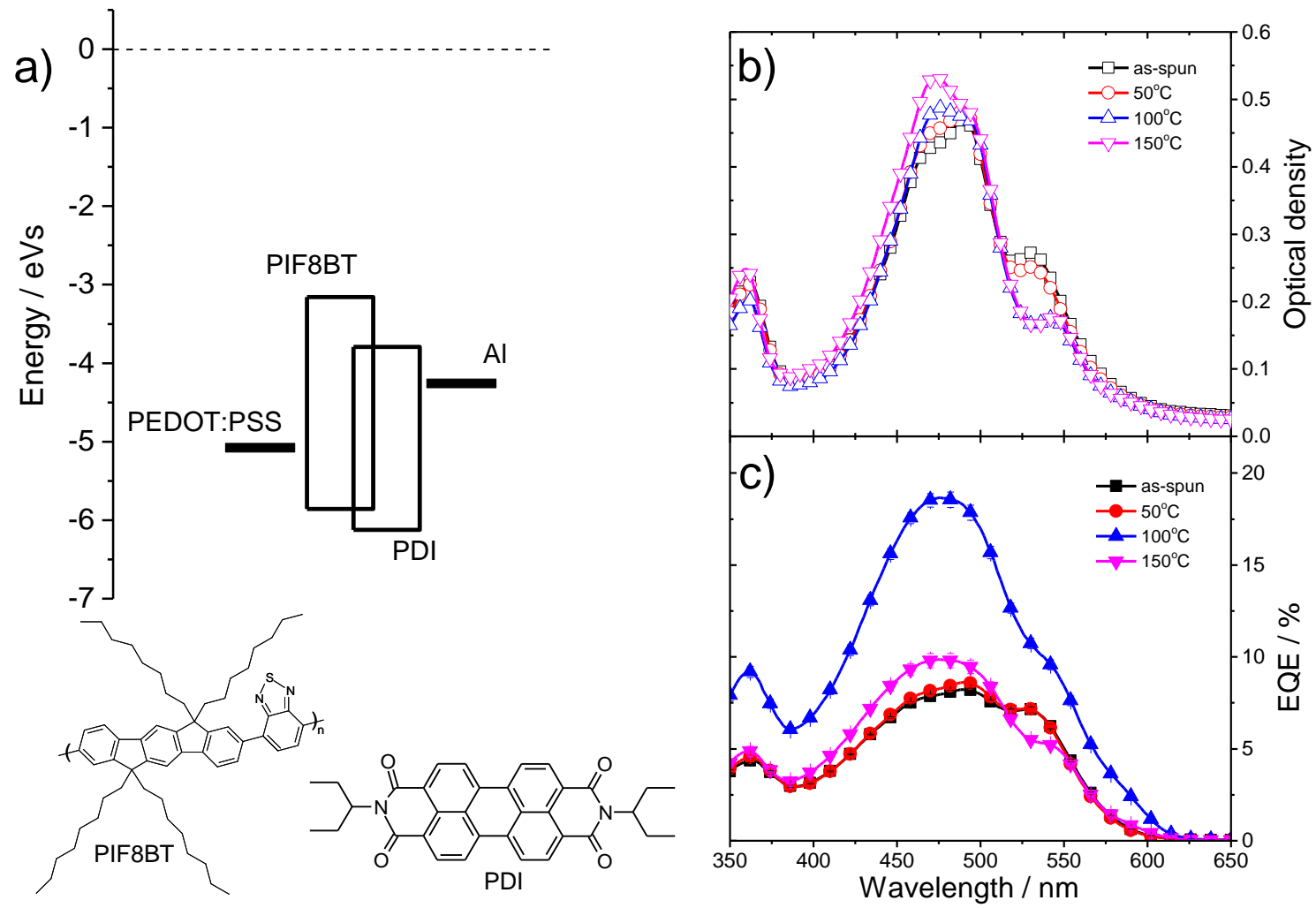

Figure 1. a) The frontier orbital alignment of the PIF8BT and PDI materials used in this study together with the work function energies of the PEDOT:PSS hole-collecting and the Al electron-collecting electrodes. b) Absorption spectra of PIF8BT:PDI blend films deposited on quartz substrates before thermal annealing (as-spun, open squares) and after thermal annealing at $50{ }^{\circ} \mathrm{C}$ (open circles), $100{ }^{\circ} \mathrm{C}$ (open up-triangles) and $150{ }^{\circ} \mathrm{C}$ (open down-triangles). c) External quantum efficiency spectra of PIF8BT:PDI photovoltaic devices with photoactive layers before thermal annealing (as-spun, filled squares) and after thermal annealing at $50{ }^{\circ} \mathrm{C}$ (filled circles), $100{ }^{\circ} \mathrm{C}$ (filled up-triangles) and $150{ }^{\circ} \mathrm{C}$ (filled down-triangles)

The UV-Vis absorption spectrum of the PIF8BT:PDI bulk heterojunction spans the $350-600 \mathrm{~nm}$ spectral range due to the superposition of the absorption spectra of the PIF8BT and PDI components alone. Notably, even at the highest annealing temperature used $\left(150^{\circ} \mathrm{C}\right)$ the characteristic absorption peak of the PDI crystallite at $590 \mathrm{~nm}^{38}$ is not detectable. Yet, the reduced absorption intensity at around $530 \mathrm{~nm}$ that is observed together with the main absorption band at around $450 \mathrm{~nm}$ verify that aggregation of the PDI component takes place following thermal annealing.

Photodiode devices of the glass/ITO/PEDOT:PSS/PIF8BT:PDI/Al geometry were fabricated and the PIF8BT:PDI photoactive layers were thermally annealed between $50-150{ }^{\circ} \mathrm{C}$. For these systems, the external quantum efficiency (EQE) spectra are shown in Figure 1b. In line with the UV-Vis spectra, 
the corresponding EQE device response verifies a substantial photocurrent generation efficiency in the spectral range of $350-600 \mathrm{~nm}$. In particular, the maximum photocurrent is produced by the device with a PIF8BT:PDI photoactive layer annealed at $100{ }^{\circ} \mathrm{C}$; a maximum EQE of $18.6 \%$ is obtained at $475 \mathrm{~nm}$ where the PDI mainly absorbs as the major component of the PIF8BT:PDI heterojunction. Steady-state PL characterization experiments (Supporting Information, Figure S1) confirm that the quenching of the PDI luminescence is gradually reduced as the thermal annealing temperature increases, ${ }^{26}, 39$ suggesting that less PDI excited states dissociate at the annealed PDI/PIF8BT interfaces. However, dark $J-V$ measurements (Supporting Information, Figure S2) performed for optimized single-carrier PIF8BT:PDI devices find that electron and hole mobility values are balanced when the PIF8BT:PDI layer is annealed at $100{ }^{\circ} \mathrm{C}$. Zero-field hole- $\left(\mu_{\mathrm{h}}\right)$ and electron- $\left(\mu_{\mathrm{e}}\right)$ mobility for each annealing temperature was deduced based on the Mott-Gurney equation by considering the Poole-Frenkel effect. ${ }^{40}$ Table 1 reports the overview of the extracted charge carrier mobility values for these systems, suggesting that the inefficient PL quenching is compensated by optimized charge transport.

Table 1. Overview of zero-field charge carrier mobility values and WAXS data

\begin{tabular}{|c|c|c|c|c|c|}
\hline $\begin{array}{c}\text { Annealing } \\
\text { temperature }\end{array}$ & $\boldsymbol{\mu}_{\mathbf{h}}\left(\mathbf{c m}^{\mathbf{2}} / \mathrm{V} \bullet \mathbf{s e c}\right)$ & $\boldsymbol{\mu}_{\mathrm{e}}\left(\mathbf{c m}^{\mathbf{2}} / \mathbf{V} \bullet \mathbf{s e c}\right)$ & $\begin{array}{c}\text { PDI } \\
\text { column } \\
\text { length } \\
(\mathbf{n m})\end{array}$ & $\begin{array}{c}\text { PDI domain } \\
\text { size (nm) }\end{array}$ & $\begin{array}{c}\text { PIF8BT } \\
\text { domain } \\
\text { size } \\
(\mathbf{n m})\end{array}$ \\
\hline As-spun & $9.8 \times 10^{-8} \pm 2.5 \times 10^{-9}$ & $1.2 \times 10^{-7} \pm 4.0 \times 10^{-9}$ & $12 \pm 2$ & $27 \pm 2$ & $6 \pm 1$ \\
\hline $50^{\circ} \mathrm{C}$ & $4.9 \times 10^{-8} \pm 5.7 \times 10^{-10}$ & $0.8 \times 10^{-7} \pm 2.8 \times 10^{-9}$ & $13 \pm 2$ & $28 \pm 2$ & $6 \pm 1$ \\
\hline $100^{\circ} \mathrm{C}$ & $3.8 \times 10^{-8} \pm 6.8 \times 10^{-10}$ & $0.9 \times 10^{-7} \pm 4.5 \times 10^{-9}$ & $16 \pm 1$ & $18 \pm 1$ & $11 \pm 1$ \\
\hline $150^{\circ} \mathrm{C}$ & $8.2 \times 10^{-6} \pm 1.1 \times 10^{-7}$ & $5.8 \times 10^{-7} \pm 2.1 \times 10^{-7}$ & $15 \pm 1$ & $18 \pm 1$ & $18 \pm 1$ \\
\hline
\end{tabular}

The photovoltaic performance of the PIF8BT:PDI system was evaluated by the electrical characterization of PIF8BT:PDI devices illuminated under simulated solar light conditions. The main device metrics of the PIF8BT:PDI devices, namely the short-circuit photocurrent density $\left(J_{s c}\right), V_{o c}$, fill factor $(F F)$ and power conversion efficiency $(P C E)$ were determined (Supporting Information Figure 
S3). Since conventional OPV device geometries with bottom PEDOT:PSS hole-collecting electrodes consistently underestimate the performance of PDI-based OPV bulk heterojunctions, ${ }^{41}$ we have additionally studied the photovoltaic performance of inverted OPV devices with the glass/ITO/ZnO/PIF8BT:PDI/ $\mathrm{V}_{2} \mathrm{O}_{5} / \mathrm{Ag}$ device configuration. Following thermal annealing, these devices performed better with an improvement in the order of $\sim 40 \%$ for $J_{s c}$ and of $\sim 15 \%$ for $V_{o c}$.

Further characterization of the inverted OPV PIF8BT:PDI devices was carried out under variable photoexcitation intensity $\left(\mathrm{I}_{\mathrm{exc}}\right)$ of continuous-wave $(\mathrm{cw})$ laser light at $532 \mathrm{~nm}$. Figure 2 presents the photoexcitation dependent response of $J_{s c}$ and the $V_{o c}$ parameters of the as-spun and annealed devices. The excitation dependence of the two parameters is best described by the functional forms $J_{s c} \propto \mathrm{I}_{\mathrm{exc}}{ }^{\beta}$ and $V_{o c} \propto s(\mathrm{kT} / \mathrm{q}) \ln \left(\mathrm{I}_{\mathrm{exc}}\right)$, respectively. In the latter, k corresponds to the Boltzmann constant, $\mathrm{T}$ to the absolute temperature and $s$ to the thermal voltage (kT/q) coefficient. ${ }^{42}$ 
a)

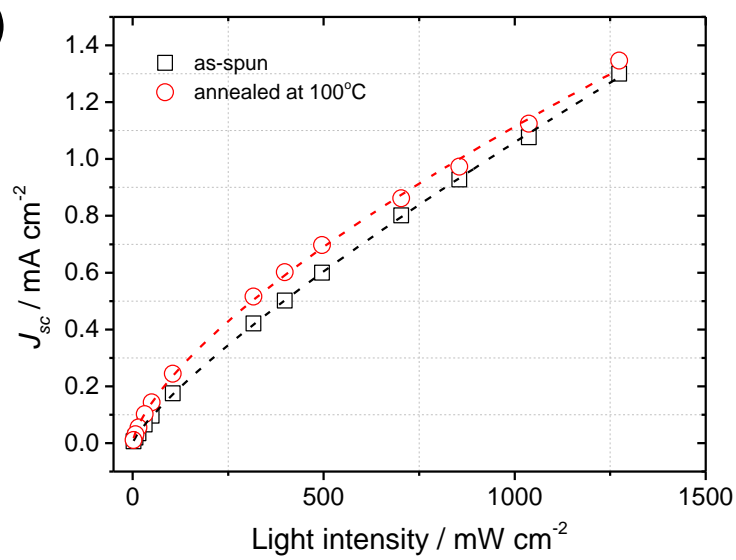

b)

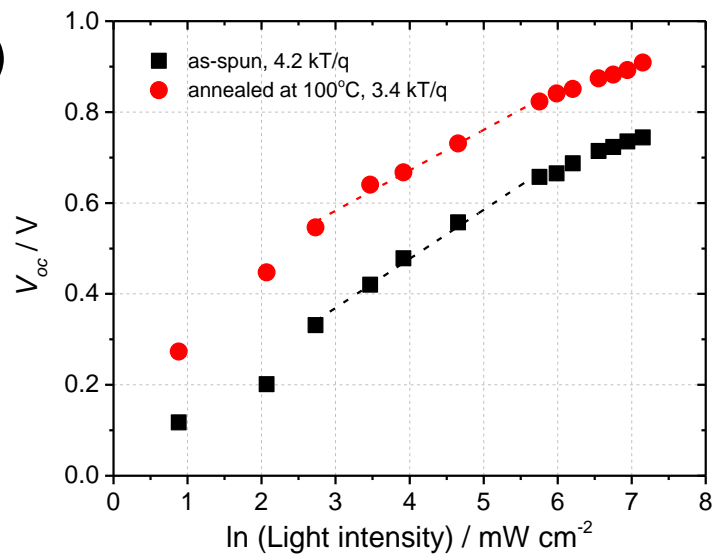

Figure 2. a) Photoexcitation-intensity dependent photocurrent density of PIF8BT:PDI inverted OPV devices with as-spun (open squares), and thermally annealed at $100{ }^{\circ} \mathrm{C}$ (open circles) photoactive PIF8BT:PDI layer. b) Photoexcitation-intensity dependent open circuit voltage of PIF8BT:PDI OPV devices with as-spun (filled squares), and thermally annealed at $100{ }^{\circ} \mathrm{C}$ (filled circles) photoactive PIF8BT:PDI layer. In both cases photoexcitation was at $532 \mathrm{~nm}$.

Although in absolute terms $J_{s c}$ increases after thermal annealing, the value of the exponent in the power-law reduces from 0.81 to 0.69 , keeping the device performance to non-optimized levels. The obtained sublinear dependence of $J_{s c}$ on $\mathrm{I}_{\mathrm{exc}}$ indicates that at short-circuit conditions either a space charge accumulates across the PIF8BT:PDI photoactive layer or that bimolecular recombination takes place in the device. ${ }^{43}$ We suggest the latter because the magnitude in the change of the hole/electron mobility ratio after thermal annealing (see in Table 1) is not as high as the change in the slope $\beta$ of the power-law. Therefore any increase in the inhomogeneous distribution of charges across the PIF8BT:PDI layer that could enhance the accumulation of space charge after thermal annealing can be ruled out. 
At $V_{o c}$ conditions the description of device operation becomes more complex. Regardless of the annealing temperature used, the obtained thermal voltage coefficients are in the range $s=3-4$; that is much higher than the typical value of $s=1$ that corresponds to free charge recombination losses. ${ }^{20,44}$ Indeed, the unusually high $s$ values imply that PIF8BT:PDI devices deviate strongly from the typical one-diode model that is accustomed for describing the operation OPV systems. In fact, high thermal voltage coefficients are endemic in a broad variety of PDI-based OPV composites prepared by many different polymeric donors (Supporting Information Figure S4). ${ }^{41}$ Disk-shaped PDI electron acceptors have the tendency to self-organize in multilength scale mesostructures. ${ }^{39}$ It is very likely this type of microstructure to contain a large concentration of physical defects that serve as electron traps. At this stage, the nature of the proposed physical defects is elusive. A careful inspection of the electron mobility values reported in Table 1 suggests that electron mobility in the PIF8BT:PDI composite is practically unaffected by thermal annealing. It remains unchanged, staying in the order of $10^{-7} \mathrm{~cm}^{2} \mathrm{~V}^{-}$

${ }^{1} \mathrm{sec}^{-1}$, and only when the PIF8BT:PDI photoactive layer is annealed at $150{ }^{\circ} \mathrm{C}$, a nearly 6 -fold increase is observed. It is plausible (see below) that the proposed defect sites are related to the disordered fraction of PDI molecules located between the ordered PDI domains, where electrons are trapped, and their transport is limited.

\section{Morphology and structure characterization}

The observed resilience of electron transport properties on thermal annealing is in line with the obtained UV-Vis results of the PIF8BT:PDI system where no substantial concentration of well-ordered PDI crystallites could be inferred. Further insight concerning the impact of thermal annealing on the structure of the PIF8BT:PDI composite in the mesoscopic and molecular length scales was gained by AFM imaging of films and WAXS experiments on extruded PIF8BT:PDI fibers, respectively.

The acquired AFM images presented in Figure 3 demonstrate that despite the apparent inertness of the charge transport properties of thermally annealed PIF8BT:PDI layers, the mesoscopic organization 
of the blend components is progressively changing with thermal annealing. Thermal processing triggers demixing of the blend components leading to a coarser separation of phases as the temperature rises from $50{ }^{\circ} \mathrm{C}$, to $100{ }^{\circ} \mathrm{C}$ and to $150{ }^{\circ} \mathrm{C}$. The typical surface texture of the PDI macro-crystals can be clearly observed in the PIF8BT:PDI films annealed at $100{ }^{\circ} \mathrm{C}$ and to $150{ }^{\circ} \mathrm{C}$, although the formation of the PDI macro-crystals is observed also in the AFM image of the PIF8BT:PDI layer annealed at 50 ${ }^{\circ} \mathrm{C}$.

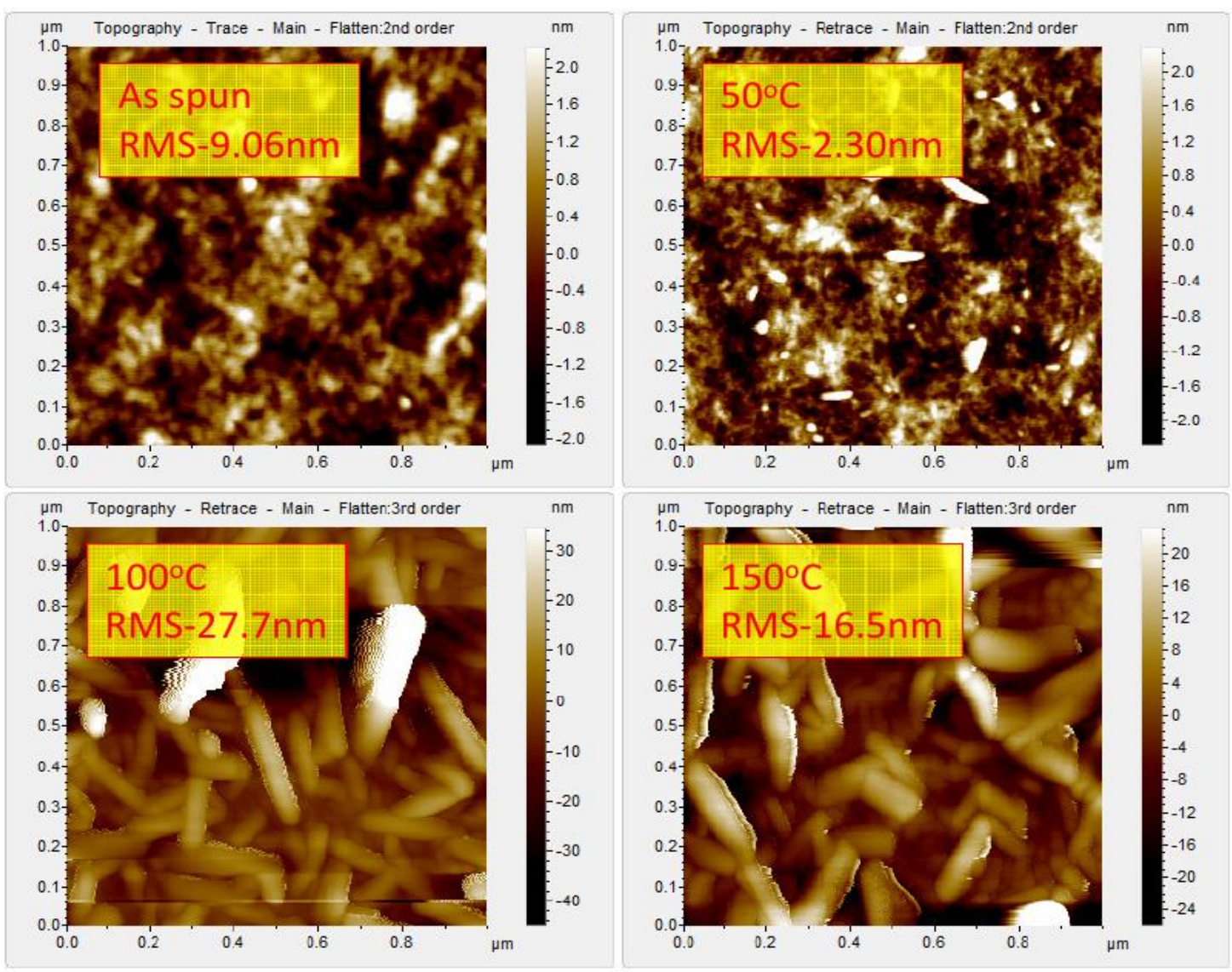

Figure 3. Atomic force microscopy images of PIF8BT:PDI blend films deposited on glass/ITO/PEDOT:PSS electrodes before thermal annealing (as-spun) and after thermal annealing at $50{ }^{\circ} \mathrm{C}$ (open circles), $100{ }^{\circ} \mathrm{C}$ (open up-triangles) and $150{ }^{\circ} \mathrm{C}$. 

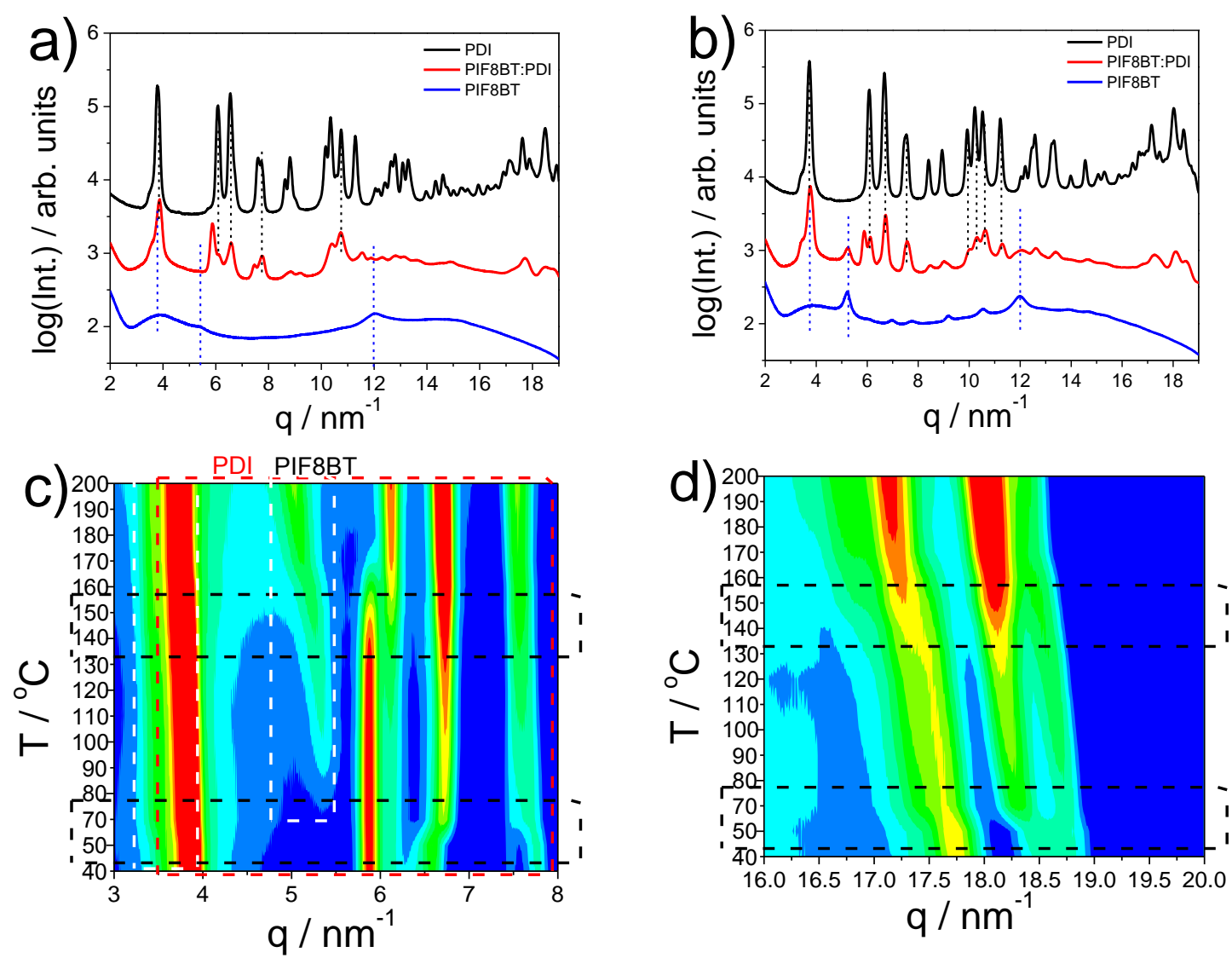

Figure 4. WAXS intensity distributions plotted as a function of the modulus of the scattering vector a) $50{ }^{\circ} \mathrm{C}$ and b) at $150{ }^{\circ} \mathrm{C}$ for PDI (black), PIF8BT (blue) and the blend PIF8BT:PDI (red). Vertical black and blue lines indicate reflections originating from PDI and PIF8BT, respectively. WAXS intensity contour plots as a function of the modulus of the scattering vector (q) for the blend PIF8BT:PDI for $q$ ranges between c) $3-8 \mathrm{~nm}^{-1}$ and d) $16-20 \mathrm{~nm}^{-1}$. Data are obtained on heating. Horizontal black rectangles indicate the temperature range of two phase transitions (see text). In c), diffraction peaks corresponding to PIF8BT and PDI are contained within the white and red rectangles, respectively.

Deeper insight on the nanostructured packing motifs of the PIF8BT:PDI composite as a function of thermal processing is gained by WAXS. The WAXS study was carried out both for the PIF8BT:PDI blend system, as well as for the PIF8BT copolymer and PDI molecular electron acceptor alone. Figure $4 \mathrm{a}, \mathrm{b}$ provide the WAXS patterns of the PDI, the PIF8BT and their blend at two temperatures. Figure $4 \mathrm{c}, \mathrm{d}$ present iso-intensity contour plots of the WAXS data as a function of the magnitude of the scattering vector (2D-WAXS intensity images are provided in Supporting Information Figure S5). The latter reveal a broad reflection at lower $q$ associated with the (100) reflection of stacked PIF8BT 
backbones (characteristic distance of $\sim 1.8 \mathrm{~nm}$ ) and an additional reflection at $q \sim 5.2 \mathrm{~nm}^{-1}$ at higher temperatures originating from the polymer with a peculiar temperature dependence. The most intense reflections, however, originate from PDI. Several other features are worth commenting. By following the PIF8BT and PDI reflections as a function of temperature two phase transformations are evident (shown by the horizontal black lines in Figure $4 \mathrm{c}$ ); one at $\sim 60^{\circ} \mathrm{C}$ (see the PDI reflection at $q \sim 6.5 \mathrm{~nm}^{-}$ ${ }^{1}$ ) revealing changes in the PDI unit cell and another at $\sim 150{ }^{\circ} \mathrm{C}$ which is common to the polymer and PDI (corresponding reflections at $\sim 5 \mathrm{~nm}^{-1}$ and $\sim 6 \mathrm{~nm}^{-1}$ ). We mention here the similarities of the temperature location of the two phase transitions with another PDI-based blend system investigated earlier by some of us. ${ }^{45}$ In addition to the location of phase transformations WAXS provides information on the PDI and polymer domains sizes as a function of temperature (extracted from the peak FWHM). The PDI domains are composed of stacks of PDI molecules in a columnar arrangement. 46 The temperature dependent WAXS measurements reveal that thermal annealing of the blend at 50 ${ }^{\circ} \mathrm{C}$ is not affecting the packing motif of the PDI and PIF8BT domains. Instead, heating the PIF8BT:PDI at $100{ }^{\circ} \mathrm{C}$ shrinks the size of the PDI domains while it doubles the domain size of the PIF8BT matrix. Annealing at $150{ }^{\circ} \mathrm{C}$ increases further the PIF8BT domain size, without affecting the size of the PDI domain. Reference WAXS measurements were performed in an identical manner when PIF8BT was replaced by poly(styrene) (PS). Owing to the amorphous nature of the PS matrix no PS domain sizes were obtained for the PS:PDI system (Figure S6 in Supporting Information). Table 1 summarizes the WAXS findings concerning the effect of thermal annealing on the PDI crystallite length, the corresponding PDI and PIF8BT domain sizes of the PIF8BT:PDI composite.

When the findings from the AFM and WAXS studies are considered together they reveal that annealing has a pronounced dependence on the length scales of phase separation as a well as on the units cells (WAXS). Particularly, the observed increase of the hole mobility at $150{ }^{\circ} \mathrm{C}$ correlates with the PIF8T phase transition observed at this temperature. 


\section{Time-integrated and time-gated photoluminescence characterization}

PL spectroscopy was employed for directly screening charge recombination losses that originate from the bulk properties of the PIF8BT:PDI composite. ${ }^{25,47}$ Figures 5a-d present a comparison between the time-integrated and the time-gated PL spectra of the PIF8BT:PDI blend films processed with different thermal annealing temperatures. The time-integrated PL acquisition (red dash lines) finds that as the annealing temperature increases from $50^{\circ} \mathrm{C}$ to $150^{\circ} \mathrm{C}$, the initially broad and featureless PDI excimer band centered at $600-650 \mathrm{~nm}$ undergoes a progressive spectral narrowing, verifying the structural reorganization of PDI aggregates to a less disordered packing motif i.e. consistent with the longer intracolumnar coherence length from WAXS (Table 1). The time-gated PL acquisition (black solid lines) enables the detection of an additional spectral feature emitting between $700-800 \mathrm{~nm}$. Considering that the PIF8BT host is not phosphorescent (Supporting Information, Figure S7), the observed signal is attributed to the delayed luminescence of the PDI/PIF8BT interfacial CT state. In agreement with the time-integrated PL data, the time-gated PL intensity ratio of the excimer/CT luminescence increases with thermal annealing. An overview of the time-gated PL spectra acquired for different delay times across the $\mu$ s time-scale is presented in Supporting Information (Figure S8), together with the time lines of the employed time-gated PL detection scheme used (Supporting Information, Scheme S1).

We have acquired the time-integrated PL spectra of the PIF8BT:PDI blends in a range of photoexcitation pulse energies. For all studied PIF8BT:PDI films the dependence of the spectrally integrated PL signals of the PDI excimer $(600-650 \mathrm{~nm})$ and the PDI/PIF8BT CT state $(700-800$ $\mathrm{nm}$ ) is shown as a function of fluence in Fig. 5e-h. Interestingly, the PL intensity of these two regions responds differently on photoexcitation intensity. In contrast to the PDI excimer luminescence, the PDI/PIF8BT CT emission exhibits a sublinear dependence on fluence, which suggests the elimination of CT species via annihilation events. ${ }^{48}$ Only when the PIF8BT:PDI blend is annealed at $150{ }^{\circ} \mathrm{C}$ a common linear response on fluence is observed for the two spectral regions, reflecting a common 
emissive state. Control measurements in reference poly(styrene) (PS):PDI blend films show that PS:PDI performs similar to the PIF8BT:PDI layer annealed at $150{ }^{\circ} \mathrm{C}$ (Supporting Information, Figure S9). The two spectral regions of the PS:PDI samples respond exhibit a comparable dependence on fuence, confirming that the PDI excimer state is the origin of the monitored luminescence in both spectral regions. 

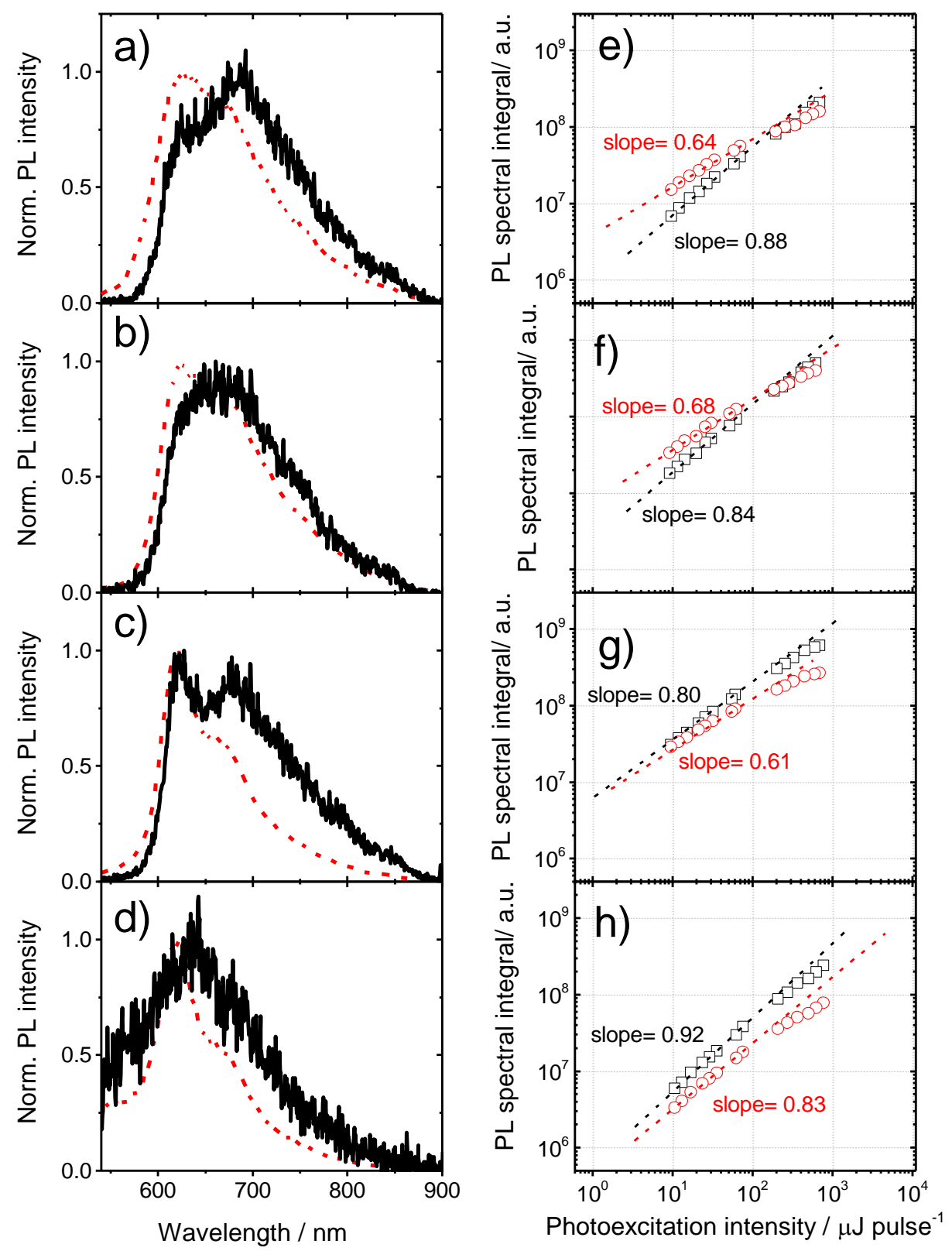

Figure 5. Normalized PL spectra of a) as-spun, b) annealed at $50{ }^{\circ} \mathrm{C}$, c) annealed at $100{ }^{\circ} \mathrm{C}$ and d) annealed at $150{ }^{\circ} \mathrm{C} \mathrm{PIF8BT:PDI} \mathrm{blend} \mathrm{films,} \mathrm{registered} \mathrm{in} \mathrm{time-integrated} \mathrm{mode} \mathrm{(dash} \mathrm{lines)} \mathrm{and} \mathrm{time-}$ gated mode; $2 \mu$ s delay time, 500 ns gate-width (solid line). Photoexcitation intensity dependent PL intensity of PDI excimer emission (squares) and PDI/PIF8BT exciplex emission (circles) of e) as-spun, f) annealed at $50{ }^{\circ} \mathrm{C}, \mathrm{g}$ ) annealed at $100{ }^{\circ} \mathrm{C}$ and $\mathrm{h}$ ) annealed at $150{ }^{\circ} \mathrm{C}$ PIF8BT:PDI blend films, as obtained from time-integrated PL spectra acquisition. In all cases photoexcitation was at $530 \mathrm{~nm}$.

We further registered the time-gated kinetics of the PDI/PIF8BT CT luminescence in the $\mu$ s time-scale, for a range of photoexcitation pulse energies (Supporting Information, Figure S10). No evidence of a 
bimolecular mechanism could be found in the observed luminescence decay. The time-gated afterglow of the PDI/PIF8BT interfacial CT state was invariant to the laser fluence for delay times as long as 5 $\mu$ after photoexcitation.

\section{Quasi time-integrated detection of delayed photoluminescence}

The observed invariance of the PDI/PIF8BT CT luminescence kinetics on fluence is in line with the intensity-dependent $V_{o c}$ response of the PIF8BT:PDI devices, which suggests the dominance of trapassisted charge recombination events. ${ }^{26,49}$ However, one cannot exclude the possibility that the overall CT luminescence signal may contain contributions from the recombination of spatially separated charges that would impart a fluence-dependent character in the observed CT kinetics.

In order to effectively capture the bimolecular fraction of the PIF8BT/PDI CT luminescence dynamics we have applied a quasi time-integrated detection scheme of delayed luminescence at progressively increased fluences of pulsed photoexcitation. After exciting the PIF8BT:PDI films with a $10 \mathrm{~ns}$ long laser pulse, the generated luminescence is registered in a series of delay steps of $50 \mathrm{~ns}$ time-intervals but with a gate window fixed to $1 \mathrm{~ms}$; that is four orders of magnitude longer than the delay times used. In that way the contribution of early-time emissive charge recombination events can be gradually rejected, thereby unravelling the reaction order of the emissive charge recombination process after the photoexcitation pulse is turned off. Figure 6a displays the schematic for the time lines of the pulse photoexcitation, the delay time and the gate-width of our experiment. 
a)
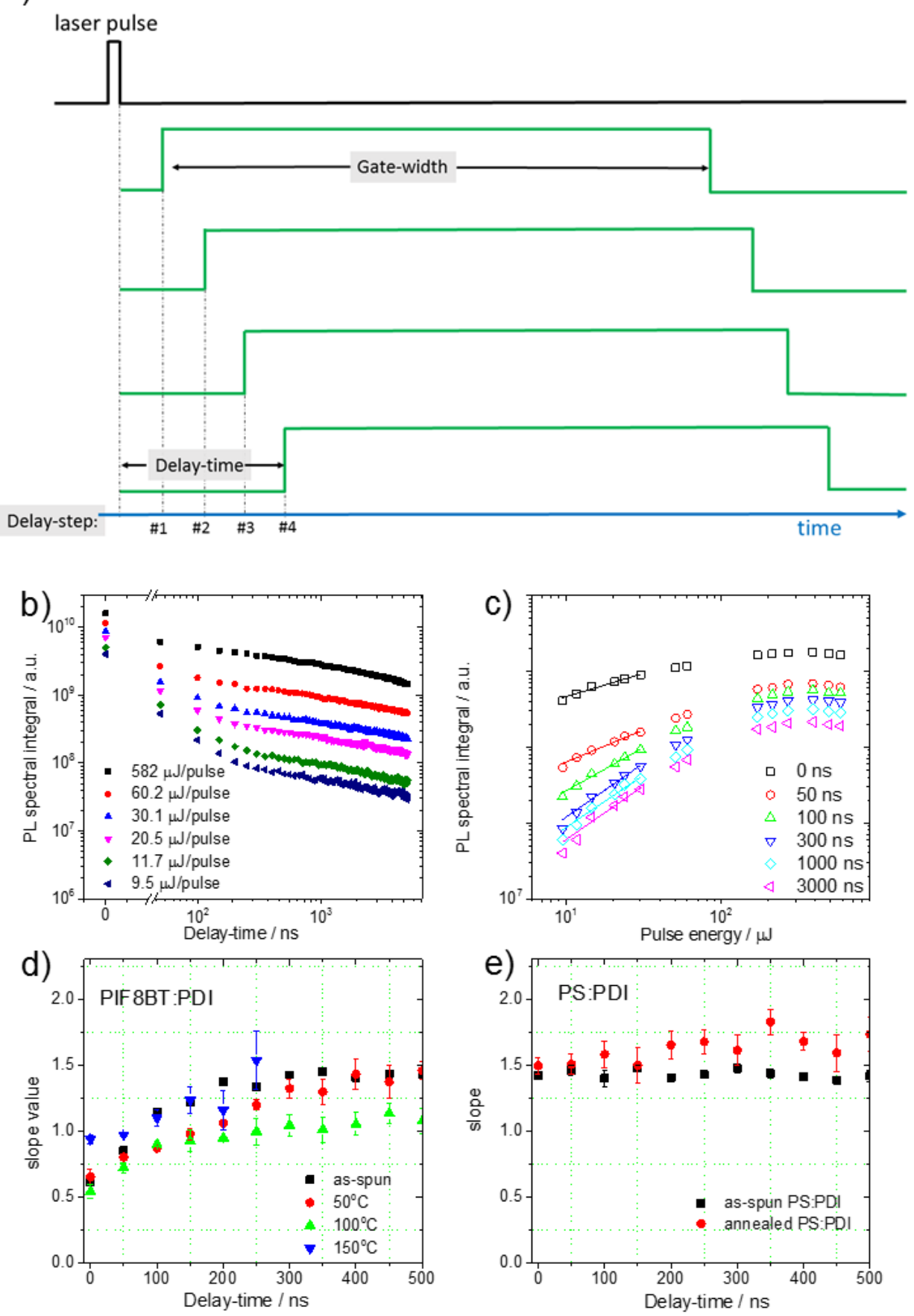

Figure 6. a) The time lines of the delay time, gate-width and the $530 \mathrm{~nm}$ laser photoexcitation pulse, employed in the quasi time-integrated detection scheme used in this work. b) Quasi time-integrated detection of the PDI/PIF8BT exciplex luminescence of the as-spun PIF8BT:PDI blend film as acquired at different laser fluences. c) Fluence-dependent quasi time-integrated detected PDI/PIF8BT exciplex luminescence of the as-spun PIF8BT:PDI blend film at different time-delays. The solid lines correspond to fits on the data with the power-law functional type of IPL $\propto \mathrm{I}_{\text {exc. }}{ }^{n}$, with $n=0.6(0 \mathrm{~ns})$, $n=0.8(50 \mathrm{~ns}), n=1.1(100 \mathrm{~ns}), n=1.4(300 \mathrm{~ns}), n=1.4(1000 \mathrm{~ns})$ and $n=1.4(3000 \mathrm{~ns})$. d) Delaydependent values of the power-law exponent $n$ used in (c) for a set of PIF8BT:PDI blend films: asspun (squares), annealed at $50{ }^{\circ} \mathrm{C}$ (circles), annealed at $100{ }^{\circ} \mathrm{C}$ (up-triangles), and annealed at $150{ }^{\circ} \mathrm{C}$ (down-triangles). e) Delay-dependent values of the power-law exponent $n$ as determined for a set of reference PS:PDI blend films: as-spun (squares) and annealed at $100{ }^{\circ} \mathrm{C}$ (circles). 
As an example, Fig.6b presents the quasi time-integrated detection of the PDI/PIF8BT CT luminescence signal of the as-spun PIF8BT:PDI blend film, as obtained for a range of photoexcitation intensities. Based on these data, the dependence of the quasi time-integrated CT luminescence on laser fluence can be tracked, and this is shown in Fig6c. Clearly, the dependence on fluence evolves with time, exhibiting an increase of the slope as the delay time between photoexcitation and detection is increased; from an initial slope value $n=0.6$ it increases to $n=1.4$ when the detection window is delayed by 3000 ns. In a similar manner we have applied the quasi time-integrated detection scheme in the whole set of PIF8BT:PDI blend films that were thermally annealed at $50{ }^{\circ} \mathrm{C}$, to $100{ }^{\circ} \mathrm{C}$ and to $150{ }^{\circ} \mathrm{C}$, thereby discerning the contributions of emissive trap-assisted and bimolecular recombination to the overall activation of PDI/PIF8BT interfacial CT states. For these films, Fig.6d presents the evolution of the slope values as the delay time between photoexcitation and detection becomes longer.

Evidently the fluence dependence of the PDI/PIF8BT CT luminescence exhibits two turnovers: (1) from sublinear to linear at $50-100 \mathrm{~ns}$ delay-times, and (2) from linear to superlinear at $100-200 \mathrm{~ns}$ delay-times. The early time regime is in good agreement with the findings of the fluence dependent time-integrated PL results of Fig.5, suggesting that CT luminescence can be quenched by both CT-CT and CT-charge annihilation interactions. The transition from the linear to superlinear regime seen in Fig.6d suggests that a fraction of the monitored PDI/PIF8BT CT afterglow is not generated by trapassisted charge recombination, but it arises by the recombination of spatially separated charges. This is not happening directly though; at relatively short delay-times, the power-law exponent is lower than one, reflecting the occurrence of annihilation events. At longer delay-times, emissive bimolecular recombination becomes important and the dependence of the interfacial PDI/PIF8BT CT luminescence on fluence becomes superlinear. Interestingly, the state of thermal annealing dictates when the fluence dependence turns from linear to superlinear. For the as-spun and the $50{ }^{\circ} \mathrm{C}$ blends slopes higher than one are obtained after $100 \mathrm{~ns}$ and $200 \mathrm{~ns}$, respectively. These times correspond to the point where a transition between trap-assisted and spatially-separated charge recombination takes place. For the 100 
${ }^{\circ} \mathrm{C}$ blend the slope remains lower than two at all times confirming that in contactless PIF8BT:PDI layer annealed at $100{ }^{\circ} \mathrm{C}$, trap-assisted charge recombination is the dominant loss recombination channel. In concert with the findings from the WAXS study this verifies that as the difference in the size of the PDI and PIF8BT domains becomes smaller, the magnitude of charge trapping is increased while emissive bimolecular charge recombination losses are reduced. This is further verified by identical measurements performed in control samples of PS:PDI blend films, both in their as-spun and annealed state. Fig.6e shows that bimolecular recombination is operative in these films: from the beginning and throughout the whole time-window of the first $500 \mathrm{~ns}$ after photoexcitation, the fluence-dependent scheme of the quasi time-integrated detection applied on the PDI luminescence exhibits a slope higher than one. Considering the findings of WAXS characterization (Figure S6 in Supporting Information) the PS:PDI blend corresponds to the largest difference between the domain size of PDI and the polymer host. As such the PS:PDI system corresponds to the extreme case where the layer order supports the occurrence of free charge recombination from the very early time after photoexcitation. At this stage the mechanism responsible for the generation of free charges in the PS:PDI system remains obscure. ${ }^{50}$ We note however that at high photoexcitation intensities auto-ionization effects caused by the annihilation of PDI excimer species is very likely to produce fully separated charges; these can then recombine for producing delayed excimer luminescence.

\section{Electro-optical device characterization}

To this end, the overview of the obtained data suggests the presence of two pools responsible for the activation of the interfacial PDI/PIF8BT CT afterglow, involving emissive trap-assisted and bimolecular recombination events. For the as-spun and $50^{\circ} \mathrm{C} \mathrm{PIF8BT:PDI} \mathrm{blends} \mathrm{the} \mathrm{difference} \mathrm{in} \mathrm{the}$ size of the PDI and PIF8BT domains is large and emissive bimolecular recombination is operative in the time window of 200 - 500 ns after photoexcitation. When the size of the PDI and PIF8BT domains layer becomes comparable, the route of emissive trap-assisted charge recombination becomes 
dominant. Similarly to the PIF8BT:PDI as-spun case, reference systems of PS:PDI blends exhibit emissive bimolecular recombination in the first $500 \mathrm{~ns}$ after photoexcitation.

In order to quantify the relative contribution of each type of charge recombination in the generation of delayed CT luminescence, we have performed electric field induced PL quenching experiments in photodiode structures with PIF8BT:PDI and PS:PDI photoactive layers. Bimolecular charge recombination is prone to external electric fields as the formation of an encounter complex of free charges at the donor/acceptor is impeded. In the presence of a static external field the probability for non-geminate charge recombination is reduced and the charges can be extracted faster. Figures 7a, b present the time-gate PL spectra of devices with annealed PS:PDI and PIF8BT:PDI photoactive layers. Evidently, the CT luminescence is quenched by the applied electric field in a reversible manner, confirming the temporal dissociation of the encounter complex when the external electric field is present. 

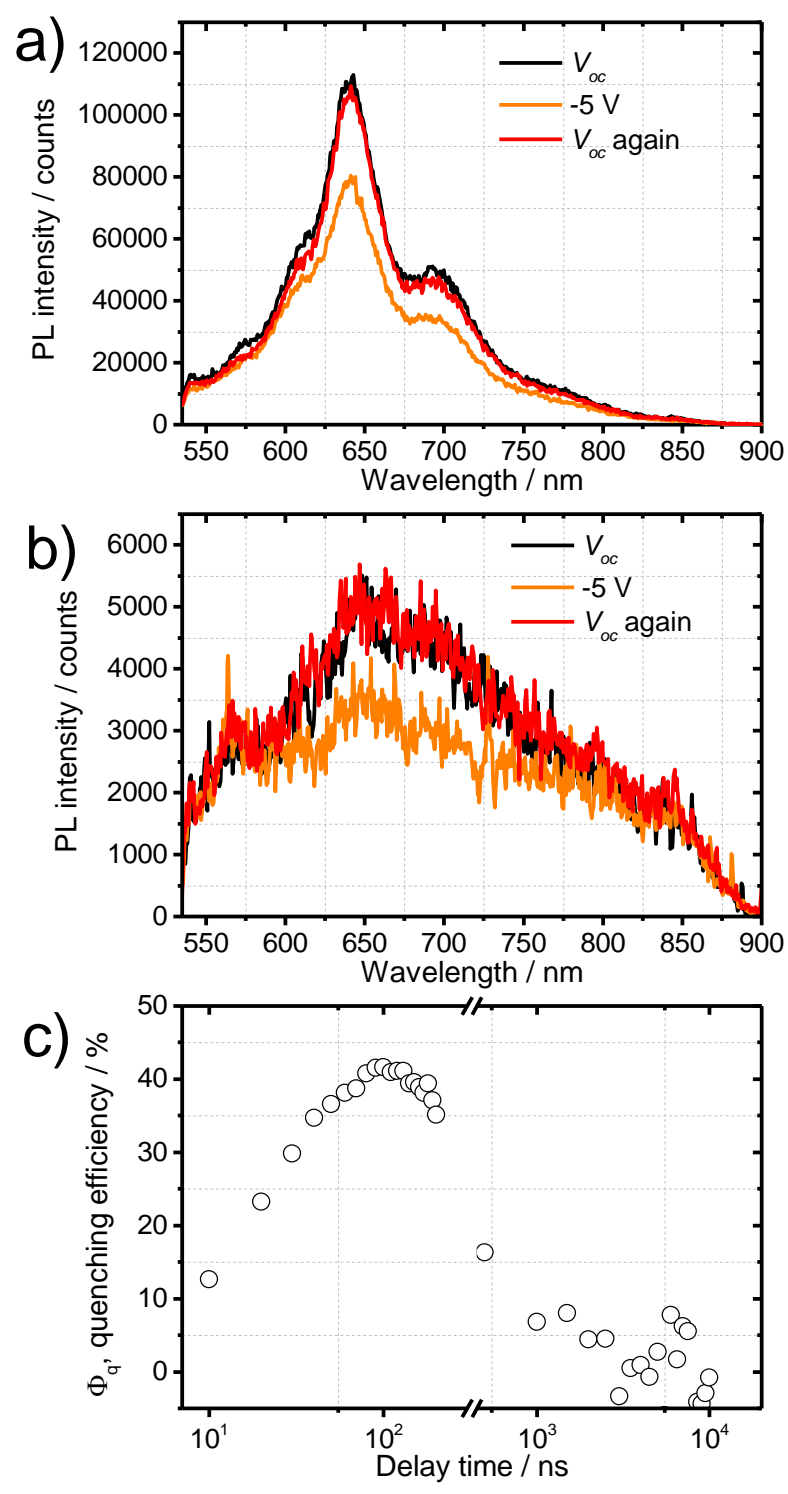

Figure 7. Time-gated PL spectra of photodiode devices photoexcited at $530 \mathrm{~nm}$ with annealed photoactive layers of a) PS:PDI (18.2 $\mu \mathrm{J}$ pulse energy, prompt PL, $10 \mathrm{~ns}$ gate-width), and b) PIF8BT:PDI (16.6 $\mu \mathrm{J}$ pulse energy, $100 \mathrm{~ns}$ delay-time, $200 \mathrm{~ns}$ gate-width) blend films. c) Electric-field induced quenching efficiency of PL signal detected in a photodiode with annealed PIF8BT:PDI layer, after the application of a $10 \mathrm{~V}$ reverse bias. In all cases the photoactive layers were annealed at 100 ${ }^{\circ} \mathrm{C}$.

Fig. $7 \mathrm{~b}$ confirms that although emissive trap-assisted recombination is the major loss channel in the annealed PIF8BT:PDI blend, a fraction of bimolecular charge recombination contributes also to the activation of the interfacial PDI/PIF8BT CT luminescence. In order to quantify the contribution of the bimolecular component, we performed a time-gated PL quenching efficiency experiment by applying a reverse bias voltage of $10 \mathrm{~V}$ on the device with a PIF8BT:PDI photoactive layer annealed $100{ }^{\circ} \mathrm{C}$. 
Figure 7c presents the quenching efficiency of the interfacial PDI/PIF8BT CT emission of this device as a function of time. The data inform that the maximum electric-field induced quenching efficiency is reached at $100 \mathrm{~ns}$ where the PDI/PIF8BT CT luminescence is quenched only by $~ 40 \%$. At longer times, in the $\mu$ s time-scale, the quenching efficiency drops to zero and the intensity of delayed CT luminescence remains unaffected by the electric field. This presents an additional confirmation that at $V_{o c}$ conditions the contribution of emissive bimolecular recombination in the afterglow of a device with PIF8BT:PDI layer annealed at $100{ }^{\circ} \mathrm{C}$, takes place within the first $100-200 \mathrm{~ns}$ albeit it corresponds to a small fraction of the overall non-recombination losses. In this time window, most charges $(\sim 60 \%)$ follow an emissive trap-assisted recombination route leading to a strong delayed PDI/PIF8BT CT luminescence that remains unquenched upon applying an external electric field. At even longer time scales (in the $\mu$ s time range), trap-assisted charge recombination becomes dominant (100\%), dictating the interfacial PDI/PIF8BT CT afterglow and imparting a photoexcitation intensity independent character on the time-gated PDI/PIF8B CT luminescence kinetics.

\section{Discussion}

The PIF8BT:PDI material combination is a rather ineffective OPV bulk heterojunction system, suffering by poor light absorption at low photon energies, low device photocurrent and enhanced nongeminate charge recombination losses. However, owing to its ability to exhibit afterglow, this composite is an ideal model system for drawing information concerning the impact of layer order on the magnitude of emissive non-geminate charge recombination. The latter can be monitored via delayed luminescence spectroscopy in the ns - $\mu$ s time scale.

The level of phase separation and degree of order within the phases of the PIF8BT:PDI blend can be accurately tuned via thermal annealing in the temperature range from ambient temperature to $150^{\circ} \mathrm{C}$. Thermal processing impacts on i) the mesoscopic level by triggering a gradual demixing of the PIF8BT and PDI components in the blend, and ii) the molecular level by a gradually balancing the size of 
ordered PDI and PIF8BT nanodomains. The findings of the WAXS study reported in Table 1 reveal that the nearly five times larger domain size of PDI obtained at ambient temperature becomes gradually comparable to the size of PIF8BT domain following annealing at $150^{\circ} \mathrm{C}$. In addition, two phase transitions, one at lower temperatures affecting the packing of PDI and another at higher temperatures affecting both the PIF8BT and PDI were identified. These details on structure are a valuable input for understanding the spectroscopic properties of the blends and the electrical properties of the corresponding OPV devices.

We found that annealing of the PIF8BT:PDI photoactive layer at $100^{\circ} \mathrm{C}$ maximizes the photocurrent generation efficiency of PIF8BT:PDI photodiodes. The counterintuitive observation of an optimum photocurrent generation efficiency in parallel with inefficient PL quenching is explained by the charge transport study in combination with the findings of the WAXS measurements. Thermal processing at $100^{\circ} \mathrm{C}$ triggers the structural optimization of the PIF8BT component in the PIF8BT:PDI blend, thereby increasing hole mobility and establishing a balanced charge transport. The severe impact of nongeminate recombination losses on the performance of the PIF8BT:PDI is evident in the photoexcitation intensity dependence of the $J_{s c}$ and $V_{o c}$ device parameters. Regardless the state of annealing, the obtained thermal voltage coefficient values are unusually high, suggesting the dominance of trapassisted charge recombination losses. What's more, the PIF8BT:PDI blend annealed at $100^{\circ} \mathrm{C}$ exhibits the strongest delayed interfacial PDI/PIF8BT CT luminescence intensity in parallel with a maximized device EQE of the corresponding device. ${ }^{26}$ In other OPV systems, ${ }^{11}$ where bimolecular recombination relaxation is the dominant loss channel, the delayed CT luminescence intensity correlates better with the device $V_{o c}$. However, in the PIF8BT:PDI heterojunction emissive bimolecular recombination is less important, yet operative.

The quasi-cw delayed detection of the interfacial PIF8BT/PDI CT afterglow at increasing photoexcitation intensities, and at different delay times provides valuable insight. The onset of emissive bimolecular recombination takes place at time delays higher than $100-200 \mathrm{~ns}$, thereby 
contributing to the PIF8BT/PDI CT afterglow. Nevertheless, the route of trap-limited charge recombination is the most effective. Electric-field induced PL quenching experiments informs about the branching of the trap-assisted and bimolecular emissive recombination routes in the PIF8BT:PDI system. For the blend annealed at $100{ }^{\circ} \mathrm{C}$, only $40 \%$ of the overall PIF8BT/PDI CT afterglow comes from the recombination of fully separated charges, whereas in the as-spun blend is increased.

Based on the deeper insight gained by WAXS, the effect of OPV layer order on the branching of the trap-assisted and bimolecular emissive recombination routes can be discussed. Thermal annealing minimizes the disparity in the domain sizes of the PDI and PIF8BT components and it suppresses the contribution of bimolecular charge recombination in the overall delayed PIF8BT/PDI CT luminescence. This finding complements previous reports concerning the impact of OPV layer microstructure on the efficiency of free charge generation. In model systems of poly(3-hexylthiophene2,5-diyl) (P3HT):[6,6]-phenyl-C61 butyric acid methyl ester (PCBM[60]) blend films, fully separated charges are produced efficiently when medium-sized ordered phases of P3HT and PCBM[60] exist in the OPV blend that prevent geminate recombination. ${ }^{13,14}$ At present we show how a comparable domain size of the OPV blend components minimizes the bimolecular charge recombination events. In its as-spun state, the PIF8BT:PDI blend film contains PDI domains with size five times bigger than the domain size of PIF8BT. For this blend the occurrence of emissive bimolecular charge recombination is found pronounced. This is in excellent agreement with previous delayed luminescence experiments obtained for the similar blend of poly(9,9'-dioctyl-fluorene-cobenzothiadiazole) (F8BT):PDI. ${ }^{25}$ In as-spun F8BT:PDI layers, the domain size of the PDI component was found four times larger than the size of the F8BT domain. ${ }^{41}$ For that system, the delayed F8BT/PDI CT afterglow was quenched by an external electric field with an efficiency higher than $50 \%$, indicating the dominance of the bimolecular charge recombination route.

The herein presented results suggest that PDI-based OPV blends are inherently limited by trap-assisted charge recombination losses. In tandem with the fact that this type of OPV devices often exhibits a 
characteristic S-shape in the corresponding current-voltage (J-V) characteristics, the employment of a multiple-diode electrical equivalent circuit as a diode model ${ }^{51}$ should be pursued. This approach would be more suited for truly unleashing the high potential of solution processable PDI-based and similarly underperforming hybrid OPV systems, ${ }^{52}$ and for enabling the engineering of a broader gamut of photovoltaic platforms. In a broader perspective, the detection of delayed luminescence via the proposed quasi time-integrated scheme promises to facilitate the systematic study of both types of fullerene-based ${ }^{49}$ and fullerene-free ${ }^{11}$ OPV composites that are capable of exhibiting CT afterglow. More importantly, the methodology has the potential to gain deep insight on the origins of the delayed emission observed in a wider material range, i.e. encompassing systems of semiconductor nanocrystal quantum dots ${ }^{53}$ and optoelectronic devices of mixed-halide perovskites. ${ }^{54}$

\section{Conclusions}

In conclusion, the ability of the PIF8BT:PDI system to exhibit delayed luminescence of interfacial CT states activated by non-geminate charge recombination was employed for demonstrating the capability of a new methodology to conduct delayed luminescence experiments. The proposed methodology relies on the fluence-dependent quasi time-integrated detection of emissive charge recombination events that take place at increasingly delay times after pulsed photoexcitation of the system under study, while it gradually rejects the contribution of other early-time emissive processes that dominate the overall luminescence signal. Under these conditions we were able to monitor the CT afterglow the PIF8BT:PDI system, and to discriminate the two different loss pathways of trap-assisted and bimolecular charge recombination processes that occur in comparable time-scales. The temporal separation of these two emissive non-geminate charge recombination loss processes is achieved by their different time-gated response on fluence. Our study offers valuable insight on the effect of OPV layer microstructure on non-geminate recombination losses. We showed that the process of emissive bimolecular recombination gains significance as the difference in the size of the electron-accepting 
and electron-donating OPV components becomes larger. Instead, emissive trap-limited recombination losses can be favoured when the domain size of the OPV blend components is comparable.

This work suggests that delayed luminescence technique can become a powerful diagnostic tool for enabling the swift screening of a broad range of OPV composites at a low cost and with a reduced experimental complexity. The proposed methodology introduces a medium level of equipment requirements, is adaptable to most device fabrication labs, and more importantly it is extendable to similar time-gated electroluminescence experiments. As such it holds the potential to contribute in the ongoing efforts of the OPV community to identify optimum material combinations that are based on next-generation material platforms such as the emerging non-fullerene-based electron acceptors.

\section{Acknowledgements}

PEK acknowledges financial support from the People Programme (Marie Curie Actions) of the European Union's Seventh Framework Programme (FP7/2007-2013) under REA grant agreement no PIEF-GA-2011 299657 DELUMOPV, and from the Cyprus University of Technology. The research reported in this publication was supported by funding from King Abdullah University of Science and Technology (KAUST). GI would like to thank PEK for hosting him in his research group during a sabbatical leave at CSNT@IIT PoliMi where the reported experimental work was performed.

\section{Supporting Information}

Time-integrated PL spectroscopic data of PS:PDI and PIF8BT:PDI blend films, dark $J-V$ curves of unipolar PIF8BT:PDI devices, solar cell characterization data of PIF8BT:PDI devices, $V_{O C^{-}}$ dependence PDI-based OPV devices on light intensity, WAXS scattering characterization data, UVVis spectra of PIF8BT-only film, time-integrated PL spectra of PIF8BT-only film at room temperature and at $77 \mathrm{~K}$, time-gated PL spectra of PIF8BT-only film at $77 \mathrm{~K}$, time-gated PL spectra of PIF8BT:PDI 
blend films, intensity-dependent PL data for PS:PDI blend films, fluence-dependent time-gated PL

kinetics of PIF8BT:PDI film. This material is supplied as Supporting Information.

\section{References}

1. Yu, G.; Gao, J.; Hummelen, J. C.; Wudl, F.; Heeger, A. J., Polymer Photovoltaic Cells: Enhanced Efficiencies via a Network of Internal Donor-Acceptor Heterojunctions. Science 1995, 270 (5243), 1789-1791.

2. Halls, J. J. M.; Walsh, C. A.; Greenham, N. C.; Marseglia, E. A.; Friend, R. H.; Moratti, S. C.; Holmes, A. B., Efficient Photodiodes from Interpenetrating Polymer Networks. Nature 1995, 376, 498. 3. Meng, L.; Zhang, Y.; Wan, X.; Li, C.; Zhang, X.; Wang, Y.; Ke, X.; Xiao, Z.; Ding, L.; Xia, R.; Yip, H.-L.; Cao, Y.; Chen, Y., Organic and Solution-Processed Tandem Solar Cells with 17.3\% Efficiency. Science 2018, 361, 1094-1098.

4. Forberich, K.; Guo, F.; Bronnbauer, C.; Brabec, C. J., Efficiency Limits and Color of Semitransparent Organic Solar Cells for Application in Building-Integrated Photovoltaics. Energy Technology 2015, 3 (10), 1051-1058.

5. Steim, R.; Ameri, T.; Schilinsky, P.; Waldauf, C.; Dennler, G.; Scharber, M.; Brabec, C. J., Organic Photovoltaics for Low Light Applications. Solar Energy Materials and Solar Cells 2011, 95 (12), 3256-3261.

6. Thostenson, J. O.; Li, Z.; Kim, C. H. J.; Ajnsztajn, A.; Parker, C. B.; Liu, J.; Peterchev, A. V.; Glass, J. T.; Goetz, S. M., Integrated Flexible Conversion Circuit between a Flexible Photovoltaic and Supercapacitors for Powering Wearable Sensors. Journal of The Electrochemical Society 2018, 165 (8), B3122-B3129.

7. Carsten, D.; Vladimir, D., Polymer-Fullerene Bulk Heterojunction Solar Cells. Reports on Progress in Physics 2010, 73 (9), 096401.

8. Clarke, T. M.; Durrant, J. R., Charge Photogeneration in Organic Solar Cells. Chemical Reviews 2010, 110 (11), 6736-6767.

9. Morteani, A. C.; Sreearunothai, P.; Herz, L. M.; Friend, R. H.; Silva, C., Exciton Regeneration at Polymeric Semiconductor Heterojunctions. Physical Review Letters 2004, 92 (24), 247402.

10. Vandewal, K.; Tvingstedt, K.; Gadisa, A.; Inganäs, O.; Manca, J. V., On the Origin of the OpenCircuit Voltage of Polymer-Fullerene Solar Cells. Nature Materials 2009, 8, 904-909.

11. Ziffer, M. E.; Jo, S. B.; Zhong, H.; Ye, L.; Liu, H.; Lin, F.; Zhang, J.; Li, X.; Ade, H. W.; Jen, A.

K. Y.; Ginger, D. S., Long-Lived, Non-Geminate, Radiative Recombination of Photogenerated Charges in a Polymer/Small-Molecule Acceptor Photovoltaic Blend. Journal of the American Chemical Society 2018, 140 (31), 9996-10008.

12. Raba, A.; Leroy, Y.; Cordan, A.-S., Organic Solar Cells: A Rigorous Model of the Donor-Acceptor Interface for Various Bulk Heterojunction Morphologies. Journal of Applied Physics 2014, 115 (5), 054508 .

13. Keivanidis, P. E.; Clarke, T. M.; Lilliu, S.; Agostinelli, T.; Macdonald, J. E.; Durrant, J. R.; Bradley, D. D. C.; Nelson, J., Dependence of Charge Separation Efficiency on Film Microstructure in Poly(3-hexylthiophene-2,5-diyl):[6,6]-Phenyl-C61 Butyric Acid Methyl Ester Blend Films. The Journal of Physical Chemistry Letters 2010, 1 (4), 734-738.

14. Howard, I. A.; Mauer, R.; Meister, M.; Laquai, F., Effect of Morphology on Ultrafast Free Carrier Generation in Polythiophene:Fullerene Organic Solar Cells. Journal of the American Chemical Society 2010, 132 (42), 14866-14876. 
15. Bakulin, A. A.; Rao, A.; Pavelyev, V. G.; van Loosdrecht, P. H. M.; Pshenichnikov, M. S.; Niedzialek, D.; Cornil, J.; Beljonne, D.; Friend, R. H., The Role of Driving Energy and Delocalized States for Charge Separation in Organic Semiconductors. Science 2012, 335 (6074), 1340-1344.

16. Vithanage, D. A.; Devižis, A.; Abramavičius, V.; Infahsaeng, Y.; Abramavičius, D.; MacKenzie, R. C. I.; Keivanidis, P. E.; Yartsev, A.; Hertel, D.; Nelson, J.; Sundström, V.; Gulbinas, V., Visualizing Charge Separation in Bulk Heterojunction Organic Solar Cells. Nat Commun 2013, 4, 2334.

17. Shuttle, C. G.; O’Regan, B.; Ballantyne, A. M.; Nelson, J.; Bradley, D. D. C.; Durrant, J. R., Bimolecular Recombination Losses in Polythiophene: Fullerene Solar Cells. Physical Review B 2008, 78 (11), 113201.

18. Maurano, A.; Hamilton, R.; Shuttle, C. G.; Ballantyne, A. M.; Nelson, J.; O’Regan, B.; Zhang, W.; McCulloch, I.; Azimi, H.; Morana, M.; Brabec, C. J.; Durrant, J. R., Recombination Dynamics as a Key Determinant of Open Circuit Voltage in Organic Bulk Heterojunction Solar Cells: A Comparison of Four Different Donor Polymers. Advanced Materials 2010, 22 (44), 4987-4992.

19. Mauer, R.; Howard, I. A.; Laquai, F., Effect of Nongeminate Recombination on Fill Factor in Polythiophene/Methanofullerene Organic Solar Cells. The Journal of Physical Chemistry Letters 2010, 1 (24), 3500-3505.

20. Proctor, C. M.; Kuik, M.; Nguyen, T.-Q., Charge Carrier Recombination in Organic Solar Cells. Progress in Polymer Science 2013, 38 (12), 1941-1960.

21. Lakhwani, G.; Rao, A.; Friend, R. H., Bimolecular Recombination in Organic Photovoltaics. Annual Review of Physical Chemistry 2014, 65 (1), 557-581.

22. MacKenzie, R. C. I.; Kirchartz, T.; Dibb, G. F. A.; Nelson, J., Modeling Nongeminate Recombination in P3HT:PCBM Solar Cells. The Journal of Physical Chemistry C 2011, 115 (19), 9806-9813.

23. Heeger, A. J., 25th Anniversary Article: Bulk Heterojunction Solar Cells: Understanding the Mechanism of Operation. Advanced Materials 2014, 26 (1), 10-28.

24. Murthy, D. H. K.; Melianas, A.; Tang, Z.; Juška, G.; Arlauskas, K.; Zhang, F.; Siebbeles, L. D. A.; Inganäs, O.; Savenije, T. J., Origin of Reduced Bimolecular Recombination in Blends of Conjugated Polymers and Fullerenes. Advanced Functional Materials 2013, 23 (34), 4262-4268.

25. Keivanidis, P. E.; Kamm, V.; Dyer-Smith, C.; Zhang, W.; Laquai, F.; McCulloch, I.; Bradley, D. D. C.; Nelson, J., Delayed Luminescence Spectroscopy of Organic Photovoltaic Binary Blend Films: Probing the Emissive Non-geminate Charge Recombination. Advanced Materials 2010, 22 (45), 51835187.

26. Keivanidis, P. E.; Kamm, V.; Zhang, W.; Floudas, G.; Laquai, F.; McCulloch, I.; Bradley, D. D. C.; Nelson, J., Correlating Emissive Non-Geminate Charge Recombination with Photocurrent Generation Efficiency in Polymer/Perylene Diimide Organic Photovoltaic Blend Films. Advanced Functional Materials 2012, 22 (11), 2318-2326.

27. Feldmann, C.; Jüstel, T.; Ronda, C. R.; Schmidt, P. J., Inorganic Luminescent Materials: 100 Years of Research and Application. Advanced Functional Materials 2003, 13 (7), 511-516.

28. Li, Y.; Gecevicius, M.; Qiu, J., Long Persistent Phosphors-From Fundamentals to Applications. Chemical Society Reviews 2016, 45 (8), 2090-2136.

29. Kabe, R.; Notsuka, N.; Yoshida, K.; Adachi, C., Afterglow Organic Light-Emitting Diode. Advanced Materials 2016, 28 (4), 655-660.

30. Xu, S.; Chen, R.; Zheng, C.; Huang, W., Excited State Modulation for Organic Afterglow: Materials and Applications. Advanced Materials 2016, 28 (45), 9920-9940.

31. Liu, Y.; MacKenzie, R. C. I.; Xu, B.; Gao, Y.; Gimeno-Fabra, M.; Grant, D.; van Loosdrecht, P. H. M.; Tian, W., Organic Semiconductors with a Charge Carrier Life Time of Over 2 Hours at Room Temperature. Journal of Materials Chemistry C 2015, 3 (47), 12260-12266.

32. Brabec, C. J., Organic Photovoltaics: Technology and Market. Solar Energy Materials and Solar Cells 2004, 83 (2), 273-292. 
33. Cowan, S. R.; Banerji, N.; Leong, W. L.; Heeger, A. J., Charge Formation, Recombination, and Sweep-Out Dynamics in Organic Solar Cells. Advanced Functional Materials 2012, 22 (6), 11161128.

34. Mauer, R.; Howard, I. A.; Laquai, F., Effect of External Bias on Nongeminate Recombination in Polythiophene/Methanofullerene Organic Solar Cells. The Journal of Physical Chemistry Letters 2011, 2 (14), 1736-1741.

35. Canning, G.; Thomas, A. K.; Dunlap, D. H.; Grey, J. K., Morphological Contributions to Interfacial Charge Trapping and Nongeminate Recombination in Polymer Solar Cells Revealed by UV Light Soaking. ACS Applied Materials \& Interfaces 2018, 10 (23), 19853-19862.

36. Athanasopoulos, S.; Greenham, N. C.; Friend, R. H.; Chepelianskii, A. D., Field-enhanced Recombination at Low Temperatures in an Organic Photovoltaic Blend. Physical Review B 2015, 92 (12), 125301.

37. Hanfland, R.; Fischer, M. A.; Brütting, W.; Würfel, U.; MacKenzie, R. C. I., The Physical Meaning Of Charge Extraction by Linearly Increasing Voltage Transients from Organic Solar Cells. Applied Physics Letters 2013, 103 (6), 063904.

38. Keivanidis, P. E.; Howard, I. A.; Friend, R. H., Intermolecular Interactions of Perylene diimides in Photovoltaic Blends of Fluorene Copolymers: Disorder Effects on Photophysical Properties, Film Morphology and Device Efficiency. Advanced Functional Materials 2008, 18 (20), 3189-3202.

39. Ye, T.; Singh, R.; Butt, H.-J.; Floudas, G.; Keivanidis, P. E., Effect of Local and Global Structural Order on the Performance of Perylene Diimide Excimeric Solar Cells. ACS Applied Materials \& Interfaces 2013, 5 (22), 11844-11857.

40. Machui, F.; Rathgeber, S.; Li, N.; Ameri, T.; Brabec, C. J., Influence of a Ternary Donor Material on the Morphology of a P3HT:PCBM Blend for Organic Photovoltaic Devices. J. Mater. Chem. 2012, 22, 15570-15577.

41. Aluicio-Sarduy, E.; Singh, R.; Kan, Z.; Ye, T.; Baidak, A.; Calloni, A.; Berti, G.; Duò, L.; Iosifidis, A.; Beaupré, S.; Leclerc, M.; Butt, H.-J.; Floudas, G.; Keivanidis, P. E., Elucidating the Impact of Molecular Packing and Device Architecture on the Performance of Nanostructured Perylene Diimide Solar Cells. ACS Applied Materials \& Interfaces 2015, 7 (16), 8687-8698.

42. Koster, L. J. A.; Mihailetchi, V. D.; Ramaker, R.; Blom, P. W. M., Light Intensity Dependence of Open-Circuit Voltage of Polymer:Fullerene Solar Cells. Applied Physics Letters 2005, 86 (12), 123509.

43. Wang, Y.; Xu, W.; Yi, J.; Zuo, C.; Gong, Y.; Liu, Y.; Lai, W.-Y.; Huang, W., Improving the Exciton Dissociation of Polymer/Fullerene Interfaces with a Minimal Loading Amount of Energy Cascading Molecular Dopant. Journal of Materials Chemistry A 2018, 6 (33), 15977-15984.

44. Gasparini, N.; Jiao, X.; Heumueller, T.; Baran, D.; Matt, G. J.; Fladischer, S.; Spiecker, E.; Ade, H.; Brabec, C. J.; Ameri, T., Designing Ternary Blend Bulk Heterojunction Solar Cells with Reduced Carrier Recombination and a Fill Factor of 77\%. Nature Energy 2016, 1, 16118.

45. Singh, R.; Shivanna, R.; Iosifidis, A.; Butt, H.-J.; Floudas, G.; Narayan, K. S.; Keivanidis, P. E., Charge versus Energy Transfer Effects in High-Performance Perylene Diimide Photovoltaic Blend Films. ACS Applied Materials \& Interfaces 2015, 7 (44), 24876-24886.

46. Tasios, N.; Grigoriadis, C.; Hansen, M. R.; Wonneberger, H.; Li, C.; Spiess, H. W.; Müllen, K.; Floudas, G., Self-Assembly, Dynamics, and Phase Transformation Kinetics of Donor-Acceptor Substituted Perylene Derivatives. Journal of the American Chemical Society 2010, 132 (21), 7478 7487.

47. Baran, D.; Li, N.; Breton, A.-C.; Osvet, A.; Ameri, T.; Leclerc, M.; Brabec, C. J., Qualitative Analysis of Bulk-Heterojunction Solar Cells without Device Fabrication: An Elegant and Contactless Method. Journal of the American Chemical Society 2014, 136 (31), 10949-10955.

48. Bell, T. D. M.; Jacob, J.; Angeles-Izquierdo, M.; Fron, E.; Nolde, F.; Hofkens, J.; Müllen, K.; Schryver, F. C. D., Charge Transfer Enhanced Annihilation Leading To Deterministic Single Photon 
Emission In Rigid Perylene End-Capped Polyphenylenes. Chemical Communications 2005, (39), 4973-4975.

49. Provencher, F.; Sakowicz, M.; Brosseau, C.-N.; Latini, G.; Beaupré, S.; Leclerc, M.; Reynolds, L. X.; Haque, S. A.; Leonelli, R.; Silva, C., Slow Geminate-Charge-Pair Recombination Dynamics at Polymer: Fullerene Heterojunctions in Efficient Organic Solar Cells. Journal of Polymer Science Part B: Polymer Physics 2012, 50 (20), 1395-1404.

50. Zhang, G.; Thomas, J. K., Spectroscopic Investigation of Photoinduced Charge Separation and Recombination in Solid Polymers. The Journal of Physical Chemistry A 1998, 102 (28), 5465-5475.

51. Steingrube, S.; Breitenstein, O.; Ramspeck, K.; Glunz S.; Schenk, A.; Altermatt, P. P., Explanation of Commonly Observed Shunt Currents in c-Si Solar Cells by Means of Recombination Statistics Beyond the Shockley-Read-Hall Approximation. J. Appl. Phys. 2011, 110, 014515-1 014515-10.

52. Gao, F.; Li, Z.; Wang, J.; Rao, A.; Howard, I. A.; Abrusci, A.; Massip, S.; McNeill, C. R.; Greenham, N. C., Trap-Induced Losses in Hybrid Photovoltaics. ACS Nano 2014, 8 (4), 3213-3221.

53. Rabouw, F. T.; Kamp, M.; van Dijk-Moes, R. J. A.; Gamelin, D. R.; Koenderink, A. F.; Meijerink, A.; Vanmaekelbergh, D., Delayed Exciton Emission and Its Relation to Blinking in CdSe Quantum Dots. Nano Letters 2015, 15, 7718-7725.

54. Feldmann, S.; Macpherson, S.; Senanayak, S. P.; Abdi-Jalebi, M.; Rivett, J. P. H.; Nan, G.; Tainter, G. D.; Doherty, T. A. S.; Frohna, K.; Ringe, E.; Friend, R. H.; Sirringhaus, H.; Saliba, M.; Beljonne, D; Stranks, S. D.; Deschler, F., Photodoping Through Local Charge Carrier Accumulation in Alloyed Hybrid Perovskites for Highly Efficient Luminescence Nature Photonics, 2019, DOI: 10.1038/s41566019-0546-8.

Table of Contents (TOC) figure

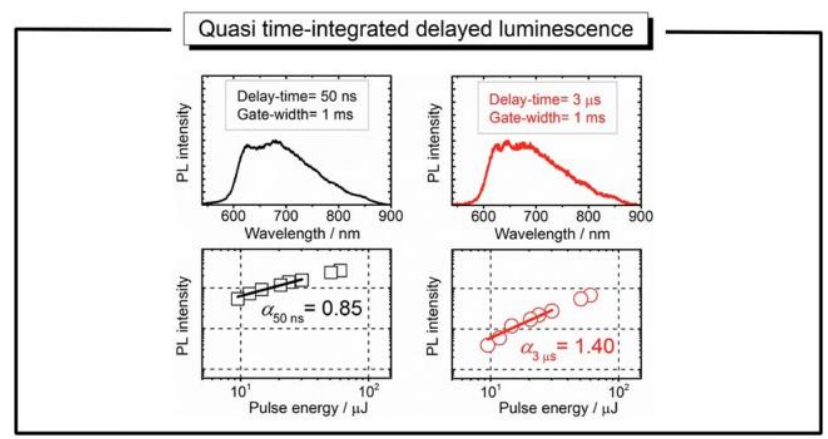

